\title{
Packet-Loss Modeling for Perceptually Optimized 3D Transmission
}

\author{
Irene Cheng, ${ }^{1}$ Lihang Ying, ${ }^{2}$ and Anup Basu ${ }^{2}$ \\ ${ }^{1}$ Department of Computer and Information Sciences, University of Pennsylvania, Philadelphia, PA 19104, USA \\ ${ }^{2}$ Department of Computing Science, University of Alberta, Edmonton, Alberta, Canada T6G $2 E 8$
}

Received 31 October 2006; Accepted 21 December 2006

Recommended by Jianfei Cai

Transmissions over unreliable networks, for example, wireless, can lead to packet loss. An area that has received limited research attention is how to tailor multimedia information taking into account the way packets are lost. We provide a brief overview of our research on designing a 3D perceptual quality metric integrating two important factors, resolution of texture and resolution of mesh, which control transmission bandwidth, followed by a suggestion on alternative strategies for packet 3D transmission of both texture and mesh. These strategies are then compared with respect to preserving 3D perceptual quality under packet loss in ad hoc wireless networks. Experiments are conducted to study how buffer size, sending rate, sending intervals, and packet size can affect loss in unreliable channels. A model for estimating the optimal packet size is then proposed. We derive the optimal number of packets based on this model, and relate the theoretical derivations to actual network data.

Copyright (c) 2007 Irene Cheng et al. This is an open access article distributed under the Creative Commons Attribution License, which permits unrestricted use, distribution, and reproduction in any medium, provided the original work is properly cited.

\section{INTRODUCTION}

An important consideration in designing effective interactive online 3D systems is to adaptively adjust the model representation, while preserving satisfactory quality as perceived by a viewer. While most research in the literature focus on geometric compression and use only synthetic texture or color, we address both geometry resolution and realistic texture resolution, and analyze how these factors affect the overall perceptual quality. Our analysis is based on experiments conducted on human observers. The perceptual quality metric derived from experiments allows the appropriate level of detail (LOD) to be selected given the computation and bandwidth constraints. Detailed surveys on simplification algorithms can be found in $[1,2]$. In order to easily control the details on a 3D object, we will follow a simple model approximation strategy based on multiresolution representation of texture and mesh. An example of geometric simplification is shown in Figure 1, in which a nutcracker-toy model is simplified to various resolution levels (number of triangles is 1260 left, 950 middle, and 538 right).

One of the major drawbacks with most 3D transmission algorithms is that they do not consider the possibility of packet loss over wireless or unreliable networks. Some wireless protocols proposed in the last decade include transmission control protocol (TCP), user datagram protocol (UDP), indirect-TCP (I-TCP) [3], and so on. For wireless networks, where packet loss occurs as a result of unreliable links and route changes, the TCP strategy leads to further delays and degradation in transmission quality. Even though issues of multimedia transmission over wireless networks have received attention [4], relatively little work has been done addressing wireless $3 \mathrm{D}$ transmission. In recent research, approaches for robust transmission of mesh over wireless networks $[5,6]$ have been outlined. However, these methods do not take joint texture and mesh transmission into account. Also, in $[5,6]$, it is assumed that some parts of the mesh can be transmitted without loss over a wireless network, allowing progressive mesh transmission to give good results. However, this assumption implies implementing a special standard with a combination of UDP and TCP protocols, which in general cannot be guaranteed in an arbitrary wireless environment. Special models for packet-loss probability have been developed by other researchers [7]. However, these models are usually associated with requirements such as retransmission. To keep our study applicable in an unrestricted ad hoc wireless environment, we simply assume packet-based transmission where a certain percentage of the packets may be lost. In this scenario, we compare how various types of $3 \mathrm{D}$ transmission strategies fare, and how to take 


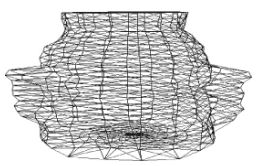

(a)

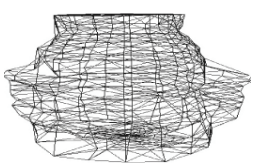

(b)

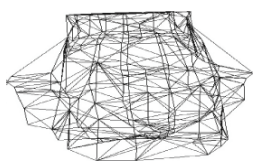

(c)
FIGURE 1: Nutcracker-toy model at various mesh resolution levels.

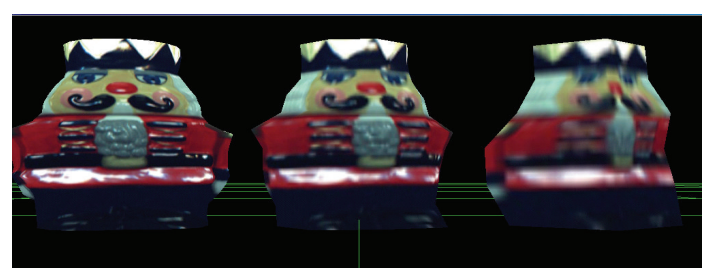

FIGURE 2: Evaluation example. perceptual quality into account in designing a better strategy.

We consider an approach based on a perceptual quality metric following our earlier work [8]. Other approaches to joint texture-mesh transmission have been discussed in $[9,10]$. The approach in [9] is based on view-dependent rate-distortion optimization, whereas our approach is viewindependent. Also, both $[9,10]$ are progressive, which necessitates greater protection of base layers in case of packet loss; our approach on the other hand does not need to guarantee delivery of certain packets in order to make other packets useful. Joint texture-mesh transmission of terrains was addressed in [11]; however, the author did not consider perceptual quality optimization.

There are two types of methods for compressing 3D meshes over lossy networks. The first approach is to compress 3D meshes in an error-resilient way. Yan et al. [12] propose to partition meshes into pieces with joint boundaries and encode each piece independently. However, if packet loss occurs, there are holes in meshes resulting from missing pieces. Jaromersky et al. [13] introduce multiple description coding for 3D meshes. Each description can be independently decoded. But it assumes the connectivity data is guaranteed to be received correctly, and is conceptually similar to Strategy A. The second set of methods use error protection to restore lost packets $[5,14]$.

Extensive research has been conducted on error resilience for audio or video communication [15-17]. Forward error correction (FEC) is a common technology to decrease the impact of packet loss. With FEC schemes, redundant data is added into the original data so that the lost original data can be recovered from the redundant data. Sending more redundant data increases the probability of recovering the loss data; however, additional redundancy increases the bandwidth requirements and the loss. The amount of redundant data should be adaptively adjusted by the characteristics of packet loss [17-21].

The remainder of this paper is organized as follows. Section 2 reviews past work on perceptual quality evaluation and discusses how to relate bandwidth with texture and mesh reduction considering perceptual quality. Section 3 examines possible strategies for $3 \mathrm{D}$ image transmission and analyzes which one is most suitable for optimizing perceptual quality under packet loss. Experimental results are presented. Different scenarios of packet loss attributed to different factors over a lossy network are presented in Section 4. A strategy for packet-size optimization is proposed in Section 5, before the work is concluded in Section 6.

\section{3D PERCEPTUAL QUALITY OPTIMIZATION}

In the area of image compression, mean square error (MSE) is commonly used as a quality predictor. However, past research has shown that MSE does not correlate well to perceived quality based on human evaluation [22]. Since this study, a number of new quality metrics based on the human visual system have been developed [23].

Several 3D objects were used as stimuli in our experiments. These objects were captured with the zoomage 3D scanner. The participants (judges) were asked to compare the target stimulus with the two referential stimuli and assign it one of the following ratings: very poor (1), poor (2), fair (3), good (4), very good (5).

Figure 2 illustrates two referential stimuli (left and right) and one target stimulus (center) in the experiment.

Considering perceptual evaluations, we observed that:

(i) perceived quality varies linearly with texture resolution (Figure 3(a));

(ii) perceived quality varies following an exponential curve for geometry (Figure 3(b)). Scaling the texture $(t)$ and geometry $(g)$ between 0 and 1 , it can be shown that

$$
\begin{aligned}
& Q(g, t) \\
& \quad=\frac{1}{1 /(m+(M-m) t)+(1 / m-1 /(m+(M-m) t))(1-g)^{c}} .
\end{aligned}
$$

Details of the perceptual evaluations and metric derivation can be found in our prior work [8]. Note that the quality value varies in the range of $1(m)$ to $5(M)$, because of the range of values allowed in the perceptual ratings.

Consider now that $b$ is the estimated total bandwidth for the transmission time interval, $T$ is the texture, and $G$ is the geometry file sizes, possibly compressed, at maximum resolution. We assume that as the texture (or geometry) is scaled by a factor $t$ (or $g$ ) in both dimensions, the corresponding file sizes get reduced to $t^{2} T$ ( or $g^{2} G$ ). To utilize the bandwidth completely, we must have

$$
b=t^{2} T+g^{2} G .
$$

Given $b$ we can choose the relative proportion of texture and mesh to create a 3D model in many different ways, as long as (2) is satisfied. The question is "What is the optimal choice maximizing perceptual quality"? Considering $m=1, M=$ 5 , and $c=2.7$ (approximately) for many objects based on 


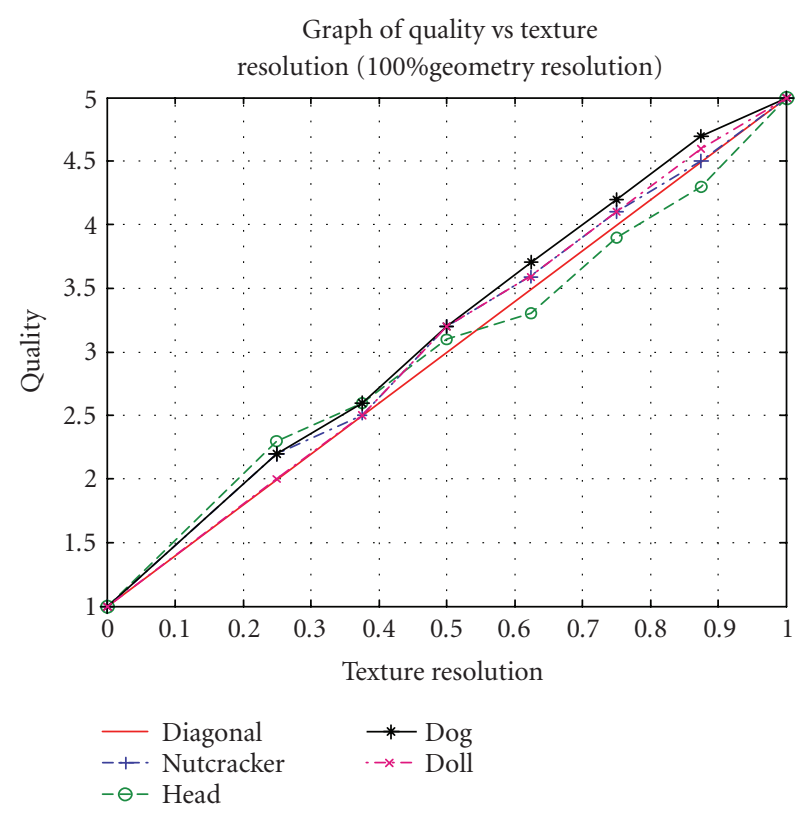

(a)

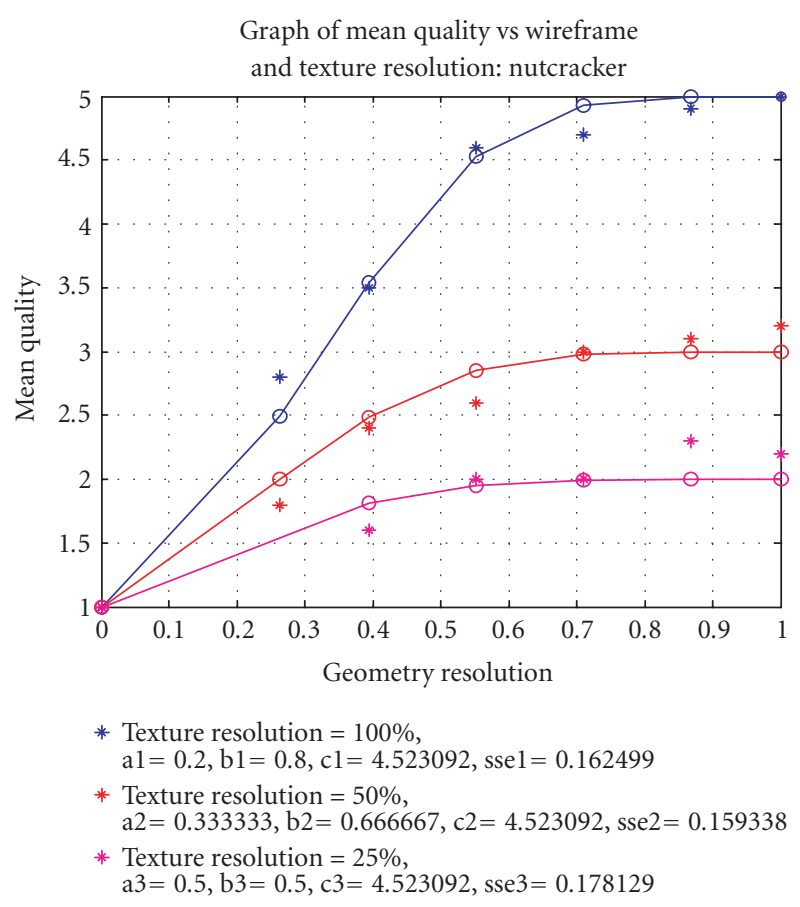

(b)

Figure 3: (a): Quality versus texture resolution (100\% geometry resolution); (b): quality versus geometry for various texture resolutions.

perceptual tests, (1) can be further simplified to

$$
Q(g, t)=\frac{1}{1 /(1+4 t)+(1-1 /(1+4 t))(1-g)^{2.7}} .
$$

Maximizing (3) is equivalent to minimizing the inverse of this equation; considering this and (2), optimizing quality reduces to minimizing

$$
Q_{b, G, T}(t)=\frac{1}{1+4 t}+\left(1-\frac{1}{1+4 t}\right)\left(1-\sqrt{\frac{b-t^{2} T}{G}}\right)^{2.7},
$$

where $b, G$, and $T$ are parameters.

Example 1. Let $b=12$ Mbits, $T=20 \mathrm{Mbits}$, and $G=$ 10 Mbits.

In this case, $t$ can only vary in the range $[\sqrt{2 / 20}$, $\sqrt{10 / 20}]=[0.316, .707]$ so that $(2)$ can be satisfied. The graph of (4) for varying $t$ for this case is shown in Figure 4. The optimal value of $t$ is close to 0.6 for this example. In general, given $T$ and $G$ for a 3D object, optimum $t$ can be precomputed for a discrete number of $b$ values in the range $[0, T+G]$.

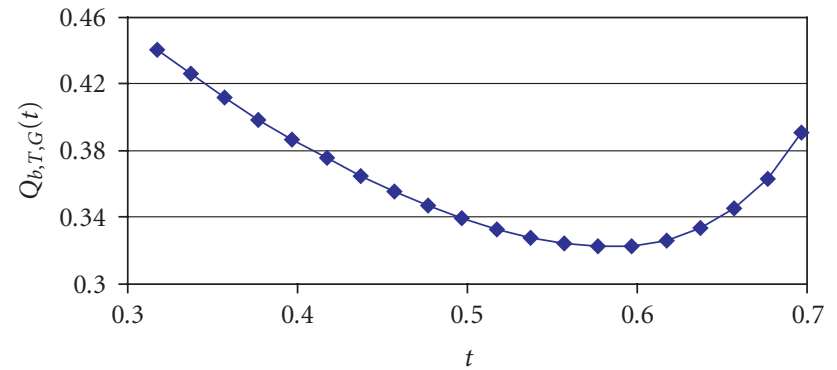

FIgURE 4: Inverse perceptual quality curve for Example 1.

\section{PERCEPTUALLY OPTIMIZED TRANSMISSION}

To simplify the model of wireless transmission, we assume that data is sent in packets of equal size and there is a possibility that a certain proportion of these packets may be lost. Various protocols [24] suggest retransmission approaches in case of packet loss; however, retransmission is not conducive to time bound real-time applications, such as $3 \mathrm{D}$ visualization for online games. We considered several possible strategies for packet construction in wireless $3 \mathrm{D}$ transmission, and then analyzed the pros and cons of each. We found that breaking up a 3D image into fragments can cause unacceptable voids; progressive transmission [25] necessitates receiving packets at lower levels before packets at higher levels can become useful; and sending duplicate copies of base layer packets in progressive transmission increases 


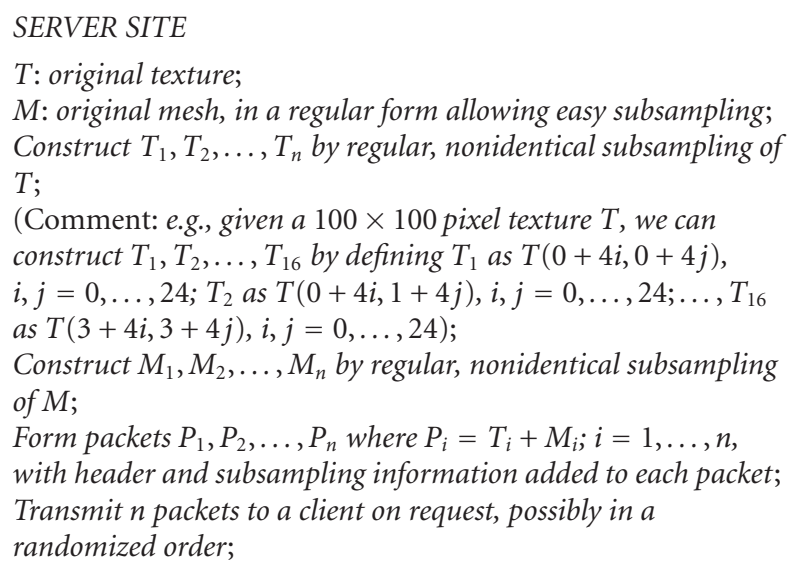

\section{CLIENT SITE}

Request server to transmit a $3 D$ object;

Receive packets from server;

Uncompress mesh and texture data stored in this packet;

Set up initial display based on first packet received and

interpolation information stored in header;

Update display based on next packet received.

\section{Algorithm 1}

bandwidth requirements. We thus focus on the two following strategies, concentrating on regular mesh transmission.

\section{Strategy $A$}

\section{$3 d$ partial information transmission}

In this approach, we break up the texture and mesh into packets by subsampling into overlapping but nonidentical components. At the client site, the overall texture and mesh are reconstructed based on interpolation from the received packets. One implementation of this approach is given Algorithm 1.

\section{Limitations of Strategy A}

One of the shortcomings of this approach is that the texture and mesh data receives equal importance; that is, the same fraction of each is transmitted in a packet. The perceptual quality analysis in the last section shows that for optimizing perceptual quality the relative importance of texture and mesh can vary depending on the available bandwidth; this issue is not taken into account in Strategy A.

\section{Strategy B}

\section{3d perceptually optimized partial information transmission}

This approach extends 3PIT by taking perceptual quality into account. The algorithm modifies Strategy A by a bandwidth

\section{SERVER SITE}

T, M: as for Strategy A;

Receive bandwidth estimate $\left(B_{e}\right)$ and estimated loss proportion (L) from requesting client;

Compute server transmitting bandwidth: $B_{s} \leftarrow B_{e} /(1-L)$; Compute optimum texture and geometry scaling factors $t_{e}$ and $g_{e}$ following procedure for minimizing $(4)$ in the last section, considering bandwidth to be $B_{e}$;

Compute scaled texture $\left(T_{s}\right)$ and mesh $\left(G_{s}\right)$, assuming transmitting bandwidth $B_{s}$, based on factors $t_{e}$ and $g_{e}$; (Comment: specifically: Ts $=\left(t_{e}^{2} /(1-L)\right) T$ and $G s=\left(g_{e}^{2} /(1-L)\right) G$; with texture and mesh possibly being interpolated to higher than the current maximum size in case the scaling factors are greater than 1);

Construct $T_{s 1}, T_{s 2}, \ldots, T_{s n}$ by regular, nonidentical subsampling of $T_{s}$;

Construct $M_{s 1}, M_{s 2}, \ldots, M_{s n}$ by regular, nonidentical subsampling of $M_{s}$;

Form packets $P_{1}, P_{2}, \ldots, P_{n}$, where $P_{i}=T_{s i}+M_{s i}$;

$i=1, \ldots, n$, with header and subsampling information added to each packet;

(Comment: number of packets $n$ is chosen based on prior decision on packet size);

Transmit $n$ packets to a client, possibly in a randomized order;

\section{CLIENT SITE}

Request server to transmit a $3 D$ object;

Receive packets from server for bandwidth estimation;

Estimate bandwidth $\left(B_{e}\right)$ based on number of packets received in a certain time interval and estimate loss proportion $(L)$; Receive packets from server containing partial data on the $3 D$ object;

Uncompress mesh and texture data stored in this packet; Set up initial display based on first packet received and interpolation information stored in header;

Update display based on next packet received.

Algorithm 2

estimation step followed by perceptually optimized packet creation. Details are described in Algorithm 2.

\section{Comments on Strategy B}

On first observation it may appear that this strategy does not take packet-loss proportion $(L)$ into account in the transmission strategy. However, in reality, this is not the case. Without any packet loss, the transmission bandwidth $\left(B_{s}\right)$ would be used to compute the optimum texture and mesh scaling factors. When packets are lost the remaining packets may not be perceptually optimal for the effective bandwidth after packet loss. We thus form packets that are optimal at a lower bandwidth $\left(B_{e}\right)$.

One of the drawbacks of Strategy B is the need to estimate bandwidth and packet loss ratio. This estimationbased transmission may not be practical where feedback from client to a server is not reliable, or for multicasting over heterogeneous networks with varying packet loss and bandwidths. This issue needs to be addressed in future research. 


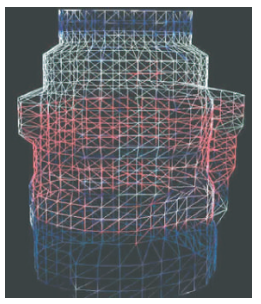

(a)

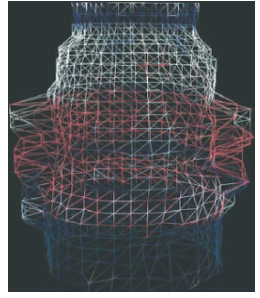

(b)

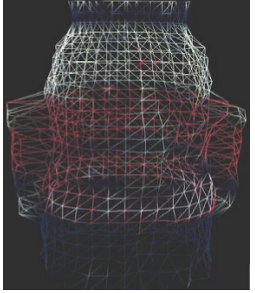

(c)

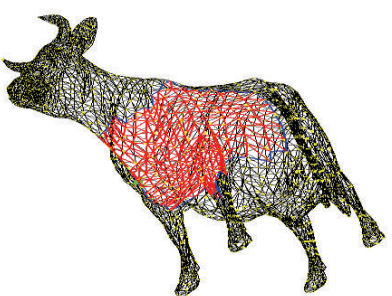

(a)

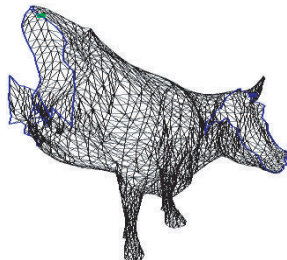

(b)
FIGURE 5: Interpolating and reconstructing mesh of nutcracker model when 2 (left), 4 (middle), and 8 of 16 packets are received.

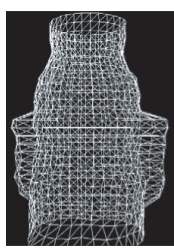

(a)

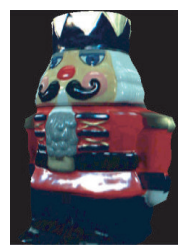

(b)

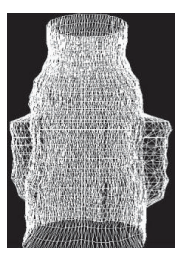

(c)

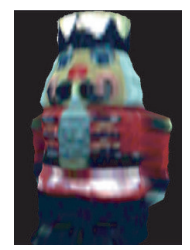

(d)
FIgURE 6: Two representations of the nutcracker texture + mesh models: left has lower quality mesh, requiring $125 \mathrm{~Kb}$ total bandwidth, but higher perceptual quality; right has higher quality mesh, resulting in lower quality texture to keep total bandwidth at $134 \mathrm{~Kb}$, but has lower perceptual quality.

\section{Experimental results of Strategy B}

We show some preliminary implementations towards deploying 3POPIT over a lossy wireless network. Figure 5 shows the effect of receiving and combining 2, 4, and 8 of 16 subsamples of the nutcracker mesh. Note that results may vary from one execution to another for a random percentage of packet loss.

Figure 6 shows the effect of optimized versus non-optimized transmission on perceptual quality. Two versions of the same model are shown, with the mesh on the left and the texture mapped on the right. Although the texture and mesh together for the left and right models use nearly the same bandwidth, 125 and $134 \mathrm{~Kb}$, respectively, the left one is favored by most viewers based on perceptual experiments.

Although a regular or semiregular mesh is used for illustration in this paper, our strategy can be extended to irregular meshes where connectivity information needs to be transmitted; triangular faces are arranged in continuous long strips following the valence driven algorithm, neighboring vertices and connectivity information are distributed evenly into different packets to minimize the risk of losing data affecting a large neighborhood [26]. Figure 7 shows how our strategy, combined with the valence driven encoding and decoding algorithm [27], can be applied to an irregular mesh.

Multiple-resolution strategy is often used to refine image or mesh data in a progressive manner. However, progressive methods $[9,10]$ necessitate greater protection of base layers in case of packet loss; our approach on the other hand does
FIgURE 7: Combining our strategy with the valence driven algorithm on an irregular cow mesh, (a) the colored patch shows the neighborhood after 400 vertices are distributed to different packets. (b) Shows the partly reconstructed mesh after 3000 vertices are retrieved from the packets.

TABLE 1: Receiving rate and packet loss for different buffer sizes.

\begin{tabular}{l|cc}
\hline Buffer size (bytes) & Receiving rate (Bps) & Packet loss (\%) \\
\hline (a) 32768 & 126611 & 0.98 \\
(b) 16384 & 127450 & 0.39 \\
(c) 4096 & 123138 & 3.91 \\
(d) 2048 & 0 & 100 \\
\hline
\end{tabular}

not need to guarantee delivery of certain packets in order to make other packets useful.

\section{NETWORK EXPERIMENTS ON PACKET LOSS}

The long-term objective of our research is to identify the appropriate parameters (packet size, sending rate, sending interval, and buffer size) for different applications to maximize throughput and minimize packet loss of UDP transmission in different Internet environments with wireless LAN access. In the experiments, the server side was a desktop computer in the Department of Computing Science, University of Alberta, Edmonton, Canada. The client was a laptop, which linked to a router following $802.11 \mathrm{~b}$. The router accessed the Internet using a cable network (with a maximum capacity of $640 \mathrm{KBps}$ ). The client was located in the same city as the server. Both client and server ran Red Hat Linux Release 9 Shrink (2.4.30 Kernel). The experiments were conducted during the day (8:00-19:00) from November 25 to November 27, 2005.

\subsection{Buffer size}

We first discuss the effect of socket buffer size on packet loss. With fixed packet size (4096 bytes) and sending rate (128 KBps), Table 1 (a) and (b) show that different buffer sizes larger than the packet size make no significant difference on packet loss. However, if buffer size is less than packet size, all packets are lost as shown in (d). The interesting point is in (c), when buffer size is equal to packet size, there is a significant packet loss as well. This can be attributed to the fluctuating bandwidth; reducing bandwidth capacity can cause an overflow on a congested buffer. In the experiments reported 


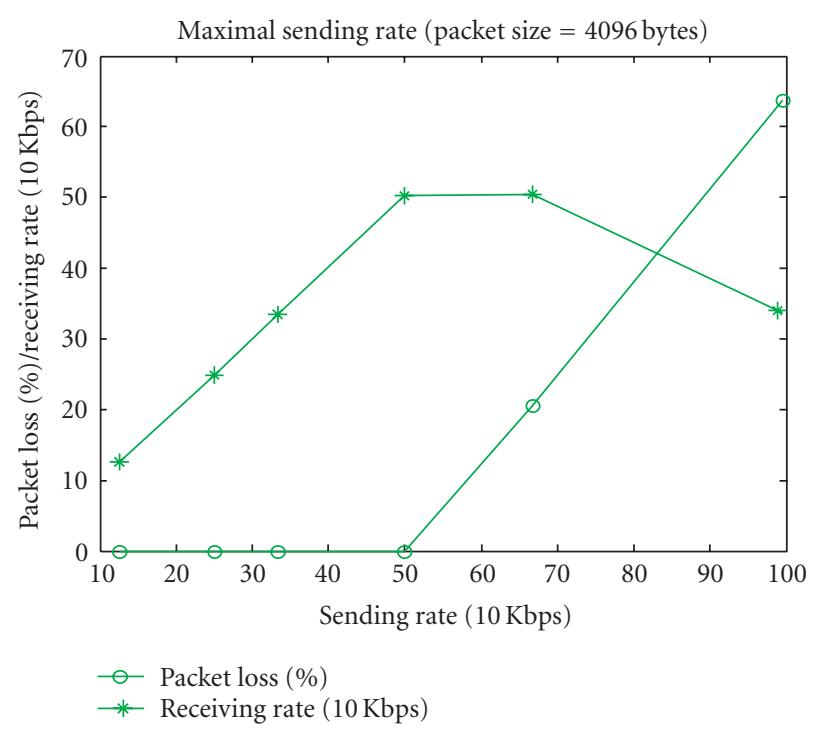

Figure 8: Effect of sending rate on packet loss.

in Sections 4.2 to 4.4 , we wanted to study packet loss independent of buffer size and therefore a large enough buffer size of 65536 was used.

\subsection{Sending rate}

Next we look into how sending rate affects packet loss. We consider a large enough sending interval and fixed packet size ( 4096 bytes), and let sending rate increase from 128 to $1024 \mathrm{Kbps}$. It can be seen from Figure 8, that as sending rate increases, receiving rate increases and packet loss remains around zero until around $500 \mathrm{Kbps}$. However, after sending rate overflows the connection (larger than $500 \mathrm{Kbps}$ ), packet loss dramatically increases and receiving rate drops owing to packet loss.

\subsection{Sending interval}

Fixing packet size at 32 bytes and without overflowing the connection, sending intervals varying from 10000,4000 , $3000,2000,1000,800,600,500$ nanoseconds were used to test the packet-loss rate. Figure 9 shows the packet loss plotted against the time interval before transmitting the next packet. Clearly, the sending interval should not be too small $(<2 \mathrm{~ms})$, otherwise loss rate can be high.

\subsection{Packet size}

Now we want to see how different packet sizes affect the receiving rate, as well as the packet loss. First, we performed experiments in an environment without other competing connections. With sending rate around $256 \mathrm{Kbps}$, without overflowing the maximum connection capacity of $640 \mathrm{Kbps}$, packet size was selected from the set of 65536,32768, $16384,8192,4096,2048,1024,512,256,128,64$, or 32 bytes. Table 2 shows that when sending interval is large enough

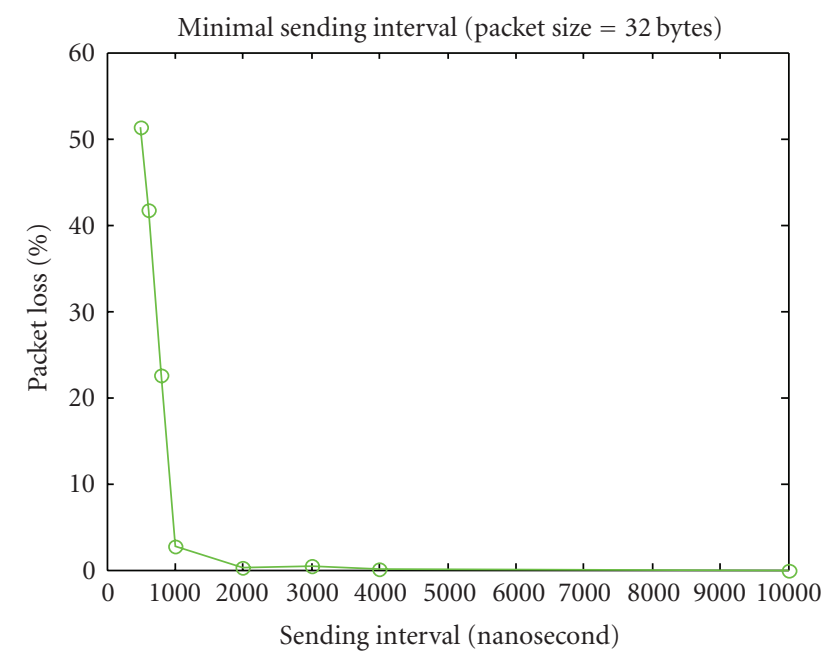

Figure 9: Packet loss versus sending interval.

TABle 2: Effect of different packet sizes on sending/receiving rate and packet loss (without competing connections).

\begin{tabular}{l|cccc}
\hline $\begin{array}{l}\text { Packet } \\
\text { size (byte) }\end{array}$ & $\begin{array}{l}\text { Sending interval } \\
\text { (nano-second) }\end{array}$ & $\begin{array}{l}\text { Sending } \\
\text { rate (Bps) }\end{array}$ & $\begin{array}{c}\text { Receiving } \\
\text { rate (Bps) }\end{array}$ & $\begin{array}{l}\text { Packet } \\
\text { loss }(\%)\end{array}$ \\
\hline 65500 & 256000 & 255510 & 253852 & 0.00 \\
32768 & 128000 & 255694 & 255694 & 0.00 \\
16384 & 64000 & 255682 & 252899 & 0.78 \\
8192 & 32000 & 255876 & 255637 & 0.00 \\
4096 & 16000 & 255690 & 252476 & 0.98 \\
2048 & 8000 & 255686 & 256480 & 0.00 \\
1024 & 4000 & 255688 & 255374 & 0.00 \\
512 & 2000 & 255724 & 255668 & 0.00 \\
256 & 1000 & 255691 & 210535 & 16.58 \\
128 & 500 & 255691 & 118407 & 53.69 \\
64 & 250 & 255682 & 485 & 99.81 \\
32 & 125 & 255401 & 499 & 99.80 \\
\hline
\end{tabular}

(equal to or larger than $2 \mathrm{~ms}$ ), different packet sizes have not much effect on sending rate, receiving rate, or packet loss. Sending rate remains stable independent of packet size. However, as mentioned above, when the sending interval is too small, packet loss sharply increases and receiving rate drops accordingly.

We then performed experiments in an environment with competing connections. In order to setup a competing environment, we configured the bandwidth of $802.11 \mathrm{~b}$ to $1 \mathrm{Mb}$. Four additional FTP concurrent connections were opened between the client and the server. Using a sending rate at $64 \mathrm{Kbps}$, packet sizes were selected from $128,256,512,1024,2048,4096,8192$, or 16384 bytes. Figure 10 shows how packet loss varies with packet size under such condition. When packet size is very small or very large, packet loss can be large. Loss was at the minimum when packet size was around 2000. 


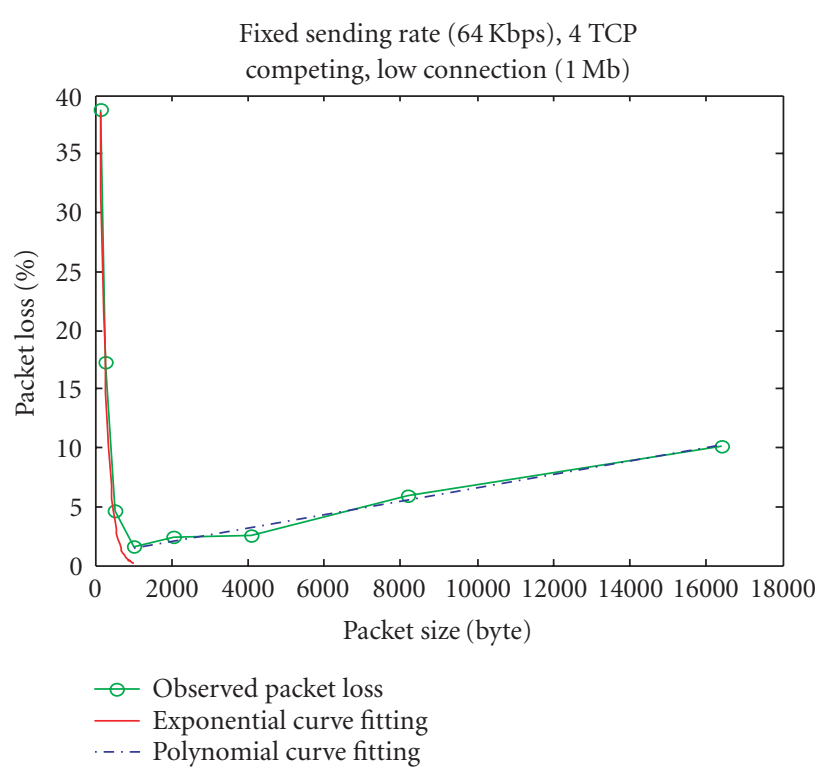

Figure 10: Packet loss versus packet size in a congested network.

In related work [28], adjusting the packet sending interval based on feedback from the network was proposed, but the work did not study the effect of different packet sizes. In [29], the authors proposed to determine the sending rate $T$ as a function of the packet size $s$, round-trip time $R$, steady-state loss event rate $\boldsymbol{p}$, and the TCP retransmit timeout value $t_{\mathrm{RTO}}$. However, they did not study how the theoretical model works in a wireless LAN (WLAN) environment. UDP throughput and CPU utility for bulk data transfer with different sender and receiver buffer sizes and packet sizes were studied in [30] but packet loss was not taken into consideration. The delay of UDP, TCP, and TCP with the option NODELAY for voice applications sending 160 bytes per 20 milliseconds was discussed in [31]. Our work differs from others by carrying out a comprehensive study on packet size, sending rate, sending interval, and buffer size through real world experiments in a competing WLAN environment, where there are packet losses besides congestion.

\section{PACKET-SIZE OPTIMIZATION}

In Figure 10, it can be observed that in the competing environment when packet size is small, the loss can be quite high because of congestion resulting from the need to route many packets. As the packet size gradually increases beyond an optimum point with low loss, the loss rate increases again. We propose a strategy to model this characteristic and determine the optimal packet size following some simplifying assumptions. We assume exponential and linear models for packet loss depending on packet size; however, the approach can be extended to other models as well. Even though various models for packet loss over wireless networks have been proposed $[4,32]$, determining the optimal packet size has not received much attention. Larger packet sizes minimize the overhead from packet headers, but have a higher probability of being corrupted. There are several studies on packet size optimization based on different metrics, such as throughput, goodput, transmission range, and energy efficiency, in wired and wireless networks [33-40].

For simplicity, we use the following assumptions and notation:

$H$ : total network header size required for each packet;

$S$ : amount of payload (application) data transmitted, if there is only 1 packet used;

$s_{n}$ : amount of payload data transmitted in each packet, if $n$ packets used; probability packet of size $D$ is not lost is

$$
e^{-\lambda D}
$$

that is, an exponential packet-loss model is used with parameter $\lambda$ and packet size as variable.

Let the amount of data transmitted be $B=S+H$ when only 1 packet is transmitted, and $s_{1}=S$. When 2 packets are transmitted, we have $B=2 s_{2}+2 H$. Since

$$
B=S+H=2 s_{2}+2 H, \quad s_{2}=\frac{S-H}{2} .
$$

In general,

$$
s_{n}=\frac{S-(n-1) H}{n} .
$$

For large $n$, that is, when $(n-1) / n \approx 1$,

$$
s_{n} \approx \frac{S}{n}-H
$$

Lemma 1. Following the model and assumptions above and independent transmission of packets, for large $n$, the total expected payload received with $n$ packets given $B=S+H$ is

$$
f(n) \approx e^{\lambda H}\left[e^{-(\lambda S) / n}(S-n H)\right] .
$$

Proof. Follows from the definitions, and noting that the total expected payload received equals the sum of individual packet sizes times the probability that it is received

$$
f(n)=e^{-\lambda D}\left[n\left(\frac{S}{n}-\frac{(n-1) H}{n}\right)\right]=e^{-\lambda(S / n-H)}(S-n H) .
$$

Since $(n-1) / n \approx 1$ for large $n$

$$
f(n) \approx e^{\lambda H}\left[e^{-(\lambda S) / n}(S-n H)\right] .
$$

Theorem 1. The number of packets $n$ optimizing the expected amount of payload transmitted for the exponential model given $B=S+H$ is an integer equal to either

$$
\left\lfloor\frac{S \sqrt{\lambda^{2} H^{2}+4 \lambda H}}{2 H}-\frac{\lambda S}{2}\right\rfloor \quad \text { or }\left\lceil\frac{S \sqrt{\lambda^{2} H^{2}+4 \lambda H}}{2 H}-\frac{\lambda S}{2}\right\rceil .
$$

Proof. Follows from optimizing the function in Lemma 1 and the fact that the number of packets is an integer. 
Now, suppose that the probability a packet of size $D$ is not lost is defined by $a-\lambda D$; that is, a linear packet loss model is used with parameter $\lambda$ and packet size as variable. This model is more meaningful in case the network characteristics follow the data in Figure 10. For this linear model; can be shown the following lemma.

Lemma 2. Following the linear model and assumptions above and independent transmission of packets, for large $n$, the total expected payload received with $n$ packets given $B=S+H$ is

$$
f(n) \approx\left[a-\lambda\left(\frac{S}{n}-H\right)\right](S-n H) .
$$

Proof. Follows from the definitions, and noting that the total expected payload received equals the sum of individual packet sizes times the probability that it is received,

$$
f(n)=(a-\lambda D)[n(S / n-(n-1) H / n)] .
$$

Since $(n-1) / n \approx 1$ for large $n$,

$$
f(n) \approx\left[a-\lambda\left(\frac{S}{n}-H\right)\right](S-n H) .
$$

Theorem 2. The number of packets optimizing the expected amount of payload transmitted for the linear model given $B=$ $S+H$ is an integer equal to either

$$
\left\lfloor S \sqrt{\frac{\lambda}{\left(a H+\lambda H^{2}\right)}}\right\rfloor \text { or }\left\lceil S \sqrt{\frac{\lambda}{\left(a H+\lambda H^{2}\right)}}\right\rceil .
$$

Proof. Follows from optimizing the function in Lemma 2 and the fact that the number of packets is an integer.

For the data in Figure 10, we can observe that for the first part the curve fits a decreasing exponential function. If we only consider this part of the curve optimum point is the rightmost point because with increasing packet size (more right on the bottom axis) the overhead from total header sizes of all packets is lower and the packet loss is also lower.

For the second part, after the minimum point of the exponential part, we can fit a linear function $y=0.8842+$ $0.0006 x$. Thus the probability of a packet not lost equals $(1-y / 100)=0.9912-0.000005683 D$, that is, $a=0.9912$ and $\lambda=0.000005683$ in Theorem 2. Given $S$ and $H$ we can determine the optimum number of packets following Theorem 2 for the linear section of the graph in Figure 10.

The network packet size in our optimization strategy is independent of the processing performed at the application level; no matter how the application data, for example, texture image and mesh information, are redistributed and segmented, the processed data, likely compress, are passed to the network as a byte stream (compressed or uncompress), which is then packed into the network packets. In our simulation, an IP header ( 8 bytes) and a UDP ( 20 bytes) are added to each network packet. 2 Mbytes application data was used in our packet loss experiment, the result of which is plotted in Figure 10. Substituting $S=2$ Mbytes and $H=28$ bytes in (16) we obtain the optimal number of packets by Theorem 2 to be either 904 or 905 corresponding to a packet size of about 2212 bytes. This shows that the optimal packet size for maximizing payload (actual multimedia data without packet headers) may not necessarily correspond to the packet size with lowest loss rate. For this experiment we assumed that the header for the multimedia data was not included in the packets. If we consider duplicating multimedia header information in packets for increased reliability under packet loss, $H$ in the formula will increase giving a lower optimal number of packets or higher optimal packet size for this example.

\section{CONCLUSIONS AND FUTURE WORK}

In this paper, we discussed factors controlling 3D image degradation and outlined an approach for estimating perceptual quality considering variations in mesh and texture resolutions. A theoretical framework for determining the relative importance of texture versus mesh was presented. An approach to optimizing perceptual quality under packet loss was then outlined. Experimental results were described to validate our approach. Finally, an approach for estimating the optimal packet size was proposed, following experimental results to collect real data on packet loss in congested wireless networks. In future work, we will extend and verify our packet size estimation method with more realistic models derived from tests over wireless networks, such as taking channel fading and burst error into consideration, to refine our assumptions. Implementations and user evaluations with handheld devices will also be conducted. We will also consider issues relating to MPEG4-3DMC compatibility [41].

\section{ACKNOWLEDGMENTS}

The support of Alberta Science and Research Authority (ASRA) and NSERC are gratefully acknowledged. Part of this work was presented in IEEE International Conference on Multimedia and Expo, 2006, in Toronto, Canada.

\section{REFERENCES}

[1] P. Heckbert and M. Garland, "Survey of polygonal surface simplification algorithms," Tech. Rep. TR97-045, Computer Science Department, Carnegie Mellon University, Pittsburgh, Pa, USA, 1997.

[2] D. Luebke, M. Reddy, J. Cohen, A. Varshney, B. Watson, and R. Huebner, Level of Detail for 3D Graphics, Morgan Kaufmann, San Francisco, Calif, USA, 2002.

[3] A. Bakre and B. R. Badrinath, "I-TCP: indirect TCP for mobile hosts," in Proceedings of the 15th International Conference on Distributed Computing Systems (ICDCS '95), pp. 136-143, Vancouver, BC, Canada, May-June 1995.

[4] D. Wu and R. Negi, "Effective capacity: a wireless link model for support of quality of service," IEEE Transactions on Wireless Communications, vol. 2, no. 4, pp. 630-643, 2003.

[5] G. Alregib, Y. Altunbasak, and J. Rossignac, "Error-resilient transmission of 3D models," ACM Transactions on Graphics, vol. 24, no. 2, pp. 182-208, 2005, Earlier version in ICASSP'02.

[6] Z. Chen, B. Bodenheimer, and J. Barnes, "Robust transmission of 3D geometry over lossy networks," in Proceeding of the 8th 
International Conference on 3D Web Technology, pp. 161-172, Saint Malo, France, March 2003.

[7] K. K. Lee and S. T. Chanson, "Packet loss probability for realtime wireless communications," IEEE Transactions on Vehicular Technology, vol. 51, no. 6, pp. 1569-1575, 2002.

[8] Y. Pan, I. Cheng, and A. Basu, "Quality metric for approximating subjective evaluation of 3-D objects," IEEE Transactions on Multimedia, vol. 7, no. 2, pp. 269-279, 2005, Short version in IEEE International Conference on Image Processing 2003.

[9] S. Yang, C.-H. Lee, and C.-C. J. Kuo, "Optimized mesh and texture multiplexing for progressive textured model transmission," in Proceedings of the 12th ACM International Conference on Multimedia, pp. 676-683, New York, NY, USA, October 2004.

[10] D. Tian and G. Al-Regib, "FQM: a fast quality measure for efficient transmission of textured 3D models," in Proceedings of the 12th ACM International Conference on Multimedia, pp. 684-691, New York, NY, USA, October 2004.

[11] L. Balmelli, "Rate-distortion optimal mesh simplification and communication," Ph.D. dissertation, Ecole Polytechnique Fédérale de Lausanne, Lausanne, Switzerland, 2001.

[12] Z. Yan, S. Kumar, and C.-C. J. Kuo, "Error-resilient coding of 3-D graphic models via adaptive mesh segmentation," IEEE Transactions on Circuits and Systems for Video Technology, vol. 11, no. 7, pp. 860-873, 2001.

[13] P. Jaromersky, X. Wu, Y.-J. Chiang, and N. Memon, "Multipledescription geometry compression for networked interactive 3D graphics," in Proceedings of 3rd International Conference on Image and Graphics (ICIG '04), pp. 468-471, Hong Kong, December 2004.

[14] G. Al-Regib and Y. Altunbasak, "An unequal error protection method for packet loss resilient 3D mesh transmission," in Proceedings of the 21st Annual Joint Conference of the IEEE Computer and Communications Societies (INFOCOM '02), vol. 2, pp. 743-752, New York, NY, USA, June 2002.

[15] Y. Wang and Q.-F. Zhu, "Error control and concealment for video communication: a review," Proceedings of the IEEE, vol. 86, no. 5, pp. 974-997, 1998.

[16] Y. Wang, S. Wenger, J. Wen, and A. K. Katsaggelos, "Review of error resilient coding techniques for real-time video communication," IEEE Signal Processing Magazine, vol. 17, no. 4, pp. 61-82, 2000.

[17] J.-C. Bolot, S. Fosse-Parisis, and D. Towsley, "Adaptive FECbased error control for Internet telephony," in Proceedings of 18th Annual Joint Conference of the IEEE Computer and Communications Societie (INFOCOM '99), vol. 3, pp. 1453-1460, New York, NY, USA, March 1999.

[18] L. Baldantoni, H. Lundqvist, and G. Karlsson, "Adaptive endto-end FEC for improving TCP performance over wireless links," in Proceedings of IEEE International Conference on Communications (ICC '04), vol. 7, pp. 4023-4027, Paris, France, June 2004.

[19] S.-W. Yuk, M.-G. Kang, B.-C. Shin, and D.-H. Cho, "An adaptive redundancy control method for erasure-code-based realtime data transmission over the Internet," IEEE Transactions on Multimedia, vol. 3, no. 3, pp. 366-374, 2001.

[20] K. Park and W. Wang, "QoS-sensitive transport of real-time MPEG video using adaptive redundancy control," Computer Communications, vol. 24, no. 1, pp. 78-92, 2001.

[21] C. H. Lin, C. H. Ke, C. K. Shieh, and N. K. Chilamkurti, "An enhanced adaptive FEC mechanism for video delivery over wireless networks," in Proceedings of International Conference on Networking and Services (ICNS '06), p. 106, Santa Clara, Calif, USA, July 2006.
[22] J. L. Mannos and D. J. Sakrison, "The effects of a visual fidelity criterion on the encoding of images," IEEE Transactions on Information Theory, vol. 20, no. 4, pp. 525-536, 1974.

[23] J. O. Limb, "Distortion criteria of the human viewer," IEEE Transactions on Systems, Man and Cybernetics, vol. 9, no. 12, pp. 778-793, 1979.

[24] R. Caceres and L. Iftode, "Improving the performance of reliable transport protocols in mobile computing environments," IEEE Journal on Selected Areas in Communications, vol. 13, no. 5, pp. 850-857, 1995.

[25] H. Hoppe, "Progressive meshes," in Proceedings of the 23rd Annual Conference on Computer Graphics (SIGGRAPH '96), pp. 99-108, New Orleans, La, USA, August 1996.

[26] I. Cheng, L. Ying, and A. Basu, "A perceptually driven model for transmission of arbitrary 3D models over unreliable networks," in Proceedings of the 3rd International Symposium on 3D Data Processing, Visualization and Transmission (3DPVT '06), Chapel Hill, NC, USA, June 2006.

[27] P. Alliez and M. Desbrun, "Valence-driven connectivity encoding for 3D meshes," Computer Graphics Forum, vol. 20, no. 3, pp. 480-489, 2001.

[28] S. Floyd, M. Handley, J. Padhye, and J. Widmer, "Equationbased congestion control for unicast applications," in Proceedings of the ACM Annual Conference of the Special Interest Group on Data Communication (SIGCOMM '00), pp. 43-56, Stockholm, Sweden, August-September 2000.

[29] A. C. Feng, A. C. Kapadia, W.-C. Feng, and G. G. Belford, "Packet spacing: an enabling mechanism for delivering multimedia content in computational grids," Journal of Supercomputing, vol. 23, no. 1, pp. 51-66, 2002.

[30] Y. Gu and R. L. Grossman, "Optimizing UDP-based protocol implementations," in Proceedings of the 3rd International Workshop on Protocols for Fast Long-Distance Networks (PFLDnet'05), Lyon, France, February 2005.

[31] X. Zhang and H. Schulzrinne, "Voice over TCP and UDP," Tech. Rep. CUCS-033-04, Department of Computer Science, Columbia University, New York, NY, USA, 2004.

[32] R. El-Azouzi and E. Altman, "A queuing analysis of packet dropping over a wireless link with retransmissions," in Proceedings of the 8th International Conference on Personal Wireless Communications (PWC '03), Venice, Italy, September 2003.

[33] V. K. M. Vadakital, M. M. Hannuksela, M. Razaei, and M. Gabbouj, "Optimal IP packet size for efficient data transmission in DVB-H," in Proceedings of the 7th Nordic Signal Processing Symposium (NORSIG '06), pp. 82-85, Reykjavik, Iceland, June 2006.

[34] Y. Sankarasubramaniam, I. F. Akyildiz, and S. W. McLaughlin, "Energy efficiency based packet size optimization in wireless sensor networks," in Proceedings of the 1st IEEE International Workshop on Sensor Network Protocols and Applications (SNPA '03), pp. 1-8, Anchorage, Alaska, USA, May 2003.

[35] I. F. Akyildiz and I. Joe, "A new ARQ protocol for wireless ATM networks," in Proceedings of the IEEE International Conference on Communications (ICC '98), vol. 2, pp. 1109-1113, Atlanta, Ga, USA, June 1998.

[36] P. Lettieri and M. B. Srivastava, "Adaptive frame length control for improving wireless link throughput, range, and energy efficiency," in Proceedings of the 17th Annual IEEE Conference on Computer Communications (INFOCOM '98), vol. 2, pp. 564571, San Francisco, Calif, USA, March 1998.

[37] E. Modiano, "An adaptive algorithm for optimizing the packet size used in wireless ARQ protocols," Wireless Networks, vol. 5, no. 4, pp. 279-286, 1999. 
[38] C. K. Siew and D. J. Goodman, "Packet data transmission over mobile radio channels," IEEE Transactions on Vehicular Technology, vol. 38, no. 2, pp. 95-101, 1989.

[39] J. D. Spragins, J. L. Hammond, and K. Pawlikowski, Telecommunications: Protocols and Design, Addison Wesley, New York, NY, USA, 1991.

[40] D. Minoli, "Optimal packet length for packet voice communication," IEEE Transactions on Communications, vol. 27, no. 3, pp. 607-611, 1979.

[41] ISO/IEC 14496-2:2000, Amendment 1, "Coding of AudioVisual Objects_-Part 2: Visual version 2", 2000. 

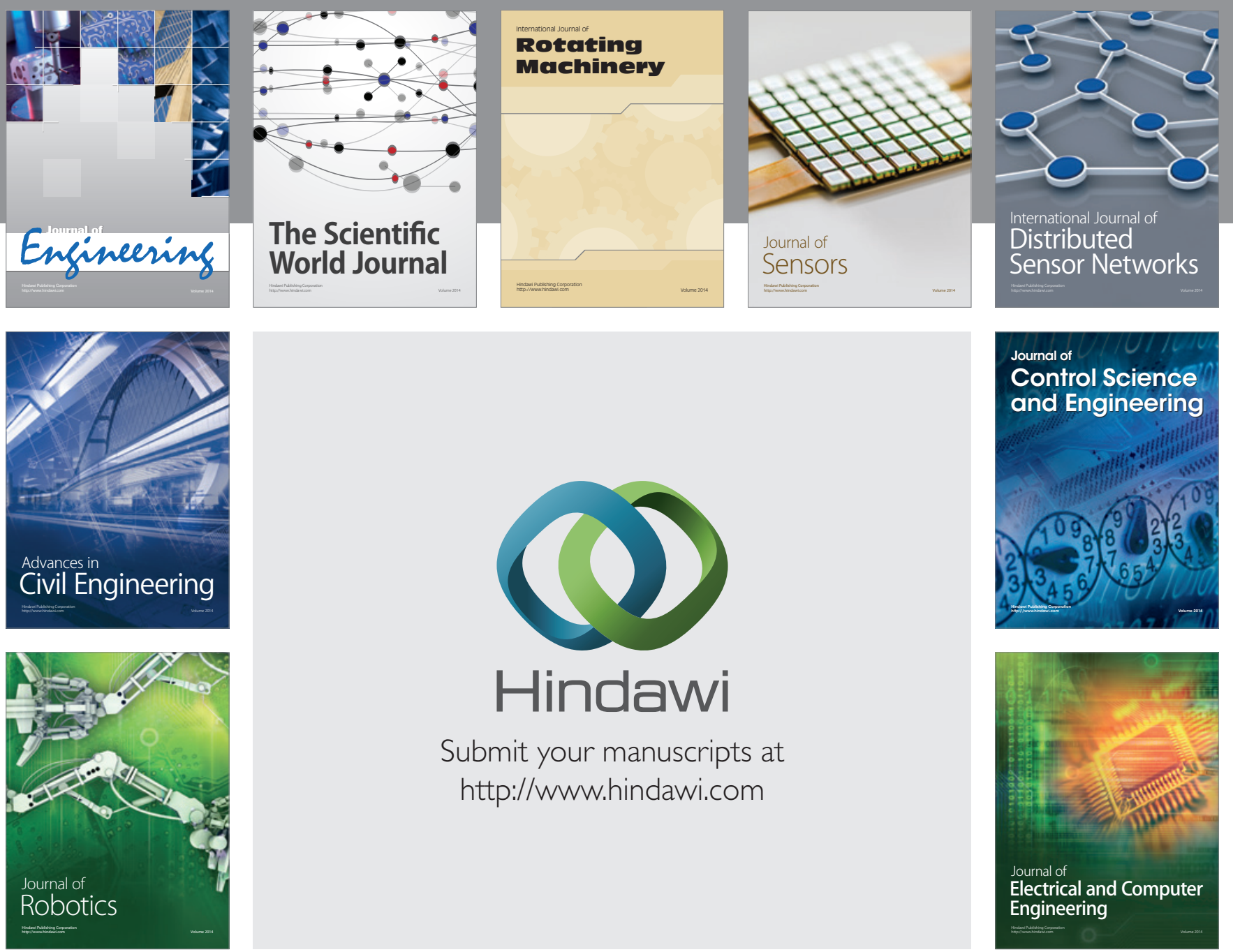

Submit your manuscripts at

http://www.hindawi.com
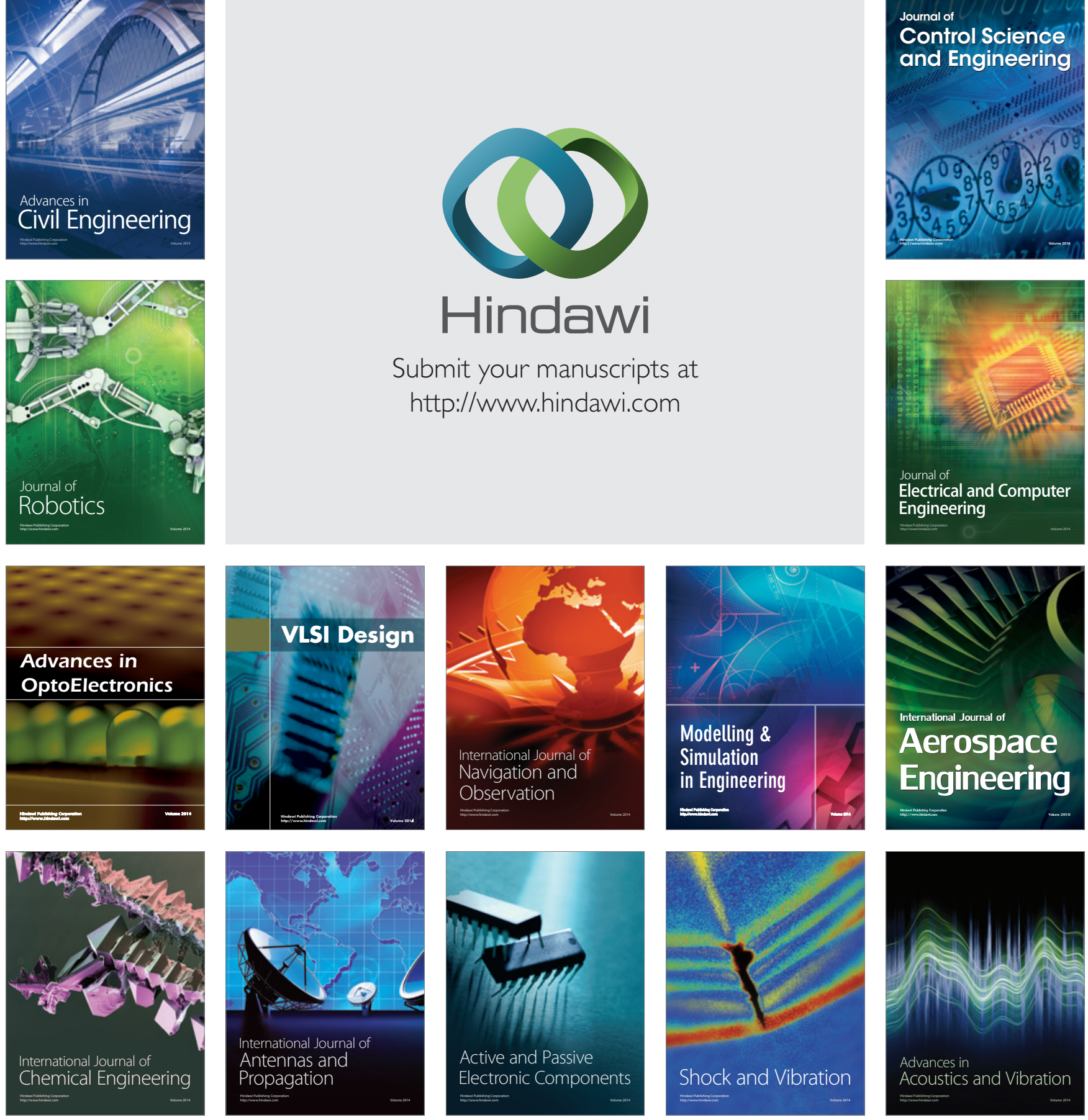